\title{
Atorvastatina Atenua o Remodelamento Vascular em Camundongos com Síndrome Metabólica
}

\author{
Atorvastatin Attenuates Vascular Remodeling in Mice with Metabolic Syndrome \\ Karine Ferreira da Silva Carvalho, ${ }^{1}$ Amanda Araújo Marques Ferreira, ${ }^{1}$ Nayara Carvalho Barbosa, ${ }^{1}$ Juliano Vilela \\ Alves, ${ }^{2}$ Rafael Menezes da Costa ${ }^{1}$ [0 \\ Universidade Federal de Jataí - Unidade Acadêmica de Ciências da Saúde, ${ }^{1}$ Jataí, GO - Brasil \\ Faculdade de Medicina de Ribeirão Preto, Universidade de São Paulo - Departamento de Farmacologia, ${ }^{2}$ Ribeirão Preto, SP - Brasil
}

\section{Resumo}

Fundamento: A síndrome metabólica é caracterizada por um conjunto de comorbidades. Durante a síndrome, observam-se alterações estruturais no sistema cardiovascular, especialmente o remodelamento vascular. Uma das causas predisponentes para essas alterações é a inflamação crônica oriunda de mudanças na estrutura e composição do tecido adiposo perivascular. Atorvastatina é eficaz no tratamento das dislipidemias. No entanto, seus efeitos pleiotrópicos não são totalmente compreendidos. Supõe-se que, durante a síndrome metabólica, ocorre remodelamento vascular e que o tratamento com atorvastatina pode ser capaz de atenuar tal condição.

Objetivos: Avaliar os efeitos do tratamento com atorvastatina sobre o remodelamento vascular em modelo experimental de síndrome metabólica.

Métodos: Camundongos Swiss receberam dieta controle ou dieta hiperglicídica por 18 semanas. Após 14 semanas de dieta, os camundongos foram tratados com veículo ou atorvastatina $(20 \mathrm{mg} / \mathrm{kg})$ durante 4 semanas. Foram avaliados o perfil nutricional e metabólico por testes bioquímicos; análise estrutural da artéria aorta por histologia e dosagem de citocinas por ensaio imunoenzimático. O nível de significância aceitável para os resultados foi p < 0,05.

Resultados: A dieta hiperglicídica promoveu o desenvolvimento de síndrome metabólica. Tal fato culminou no remodelamento hipertrófico do músculo liso vascular e tecido adiposo perivascular. Além disso, houve aumentos das citocinas TNF- $\alpha$ e IL-6 circulantes e no tecido adiposo perivascular. O tratamento com atorvastatina reduziu significativamente os danos metabólicos, o remodelamento vascular e os níveis de citocinas.

Conclusão: Atorvastatina ameniza danos metabólicos associados à síndrome metabólica induzida por dieta hiperglicídica, além de atenuar o remodelamento vascular, sendo esses efeitos associados à redução de citocinas pró-inflamatórias.

Palavras-chave: Dislipidemias; Carbohidratos da Dieta; Síndrome Metabólica; Remodelamento Vascular; Atorvastatina; Inflamação; Camundongo.

Abstract

Background: Metabolic syndrome is characterized by an array of comorbidities. During this syndrome, structural changes are observed in the cardiovascular system, especially vascular remodeling. One of the predisposing causes for these changes is chronic inflammation resulting from changes in the structure and composition of perivascular adipose tissue. Atorvastatin is effective in the treatment of dyslipidemias. However, its pleiotropic effects have not been completely understood. We hypothesize that metabolic syndrome may lead to vascular remodeling and that atorvastatin therapy may be able to attenuate this condition.

Objectives: To assess the effects of atorvastatin therapy on vascular remodeling in an experimental model of metabolic syndrome.

Methods: Swiss mice received a control diet or a hyperglicemic diet for 18 weeks. After 14 weeks of diet, mice were treated with vehicle or atorvastatin $(20 \mathrm{mg} / \mathrm{kg}$ ) during for 4 weeks. Nutritional and metabolic profiles were assessed by biochemical tests; moreover, a histological assessment of aorta structure was conducted, and cytokine levels were determined by the immunoenzyme assay. The acceptable level of significance for the results was set at $p<0.05$.

Results: Hyperglicemic diet promoted the development of metabolic syndrome. It indeed culminated in hypertrophic remodeling of vascular smooth muscle and perivascular adipose tissue. Furthermore, there were increases in the levels of circulating TNF- $\alpha$ and IL-6 and in the perivascular adipose tissue. Atorvastatin therapy significantly reduced metabolic damages, vascular remodeling, and cytokine levels.

Conclusion: Atorvastatin attenuate metabolic damages associated with metabolic syndrome induced by hyperglycemic diet, in addition to attenuating vascular remodeling; both effects are associated with reduced levels of pro-inflammatory cytokines.

Keywords: Dyslipidemias; Dietary Carbohydrates; Metabolic Syndrome; Vascular Remodeling; Atorvastatin; Inflammation; Mice.

Full texts in English - http://www.arquivosonline.com.br

Correspondência: Rafael Menezes da Costa •

Universidade Federal de Jataí - Unidade Acadêmica de Ciências da Saúde - BR 364, km 195, 3800. CEP 75801-615, Jataí, GO - Brasil

E-mail: rafael.menezess@yahoo.com.br

Artigo recebido em 13/04/2020, revisado em 14/09/2020, aceito em 04/11/2020

DOI: https://doi.org/10.36660/abc.20200322 


\section{Artigo Original}

\section{Introdução}

As doenças cardiovasculares representam grandes riscos para a qualidade de vida da população no mundo. No Brasil, aproximadamente 350 mil indivíduos morrem por doenças cardiovasculares anualmente..$^{1,2}$ Dentre as diversas causas predisponentes para riscos cardiovasculares, destaca-se a síndrome metabólica (SM), caracterizada por um conjunto de comorbidades que envolvem aumento da circunferência abdominal ( $\geq 89 \mathrm{~cm}$ para mulheres e $\geq 102 \mathrm{~cm}$ para homens), aumento nos níveis de triglicerídeos ( $\geq 150 \mathrm{mg} / \mathrm{dL}$ ), redução nos níveis de colesterol HDL $(\leq 50 \mathrm{mg} / \mathrm{dL}$ para mulheres e $\leq 40 \mathrm{mg} / \mathrm{dL}$ para homens), aumento nos níveis pressóricos $(\geq 130 / 85 \mathrm{mmHg})$ e aumento na glicemia de jejum $(\geq 100 \mathrm{mg} / \mathrm{dL})$. Dentre estas, a presença de pelo menos três comorbidades é elementar para o diagnóstico de SM. ${ }^{3,4}$

Durante a SM, observam-se alterações estruturais e funcionais dos componentes do sistema vascular. ${ }^{5-7}$ Em indivíduos com SM, há disfunção endotelial, além de aumento na migração e proliferação de células musculares lisas. Observa-se também a expansão do tecido adiposo perivascular (PVAT), evidenciada pelo aumento morfológico dos adipócitos, bem como a substituição do tecido adiposo marrom pelo tecido adiposo branco, resultando na diminuição da liberação de fatores relaxantes derivados do PVAT e consequente perda da sua capacidade de ação anticontrátil. ${ }^{8-11}$

A hipertrofia e a substituição dos adipócitos no PVAT são capazes de favorecer a produção e o acúmulo de citocinas pró-inflamatórias. ${ }^{12}$ Roedores submetidos à dieta hipercalórica e que desenvolveram SM mostraram aumento e acúmulo do PVAT nas artérias aorta e carótida. Tal fato promoveu a liberação de quimiocinas e recrutamento de monócitos e células T para o PVAT. ${ }^{13}$ Neste ambiente inflamatório, também é possível observar o aumento na produção de interleucina-6 (IL-6) e o infiltrado de macrófagos secretores do fator de necrose tumoral alfa (TNF- $\alpha$ ) no PVAT. ${ }^{14}$ Este conjunto desencadeia a inflamação crônica do sistema vascular, prejudicando a estrutura e a função dos seus constituintes.

A inflamação dos vasos sanguíneos, de fato, contribui para o remodelamento vascular, aumento da resistência periférica e desordens circulatórias. ${ }^{7,15,16}$ Neste contexto, o remodelamento vascular é um processo crônico adaptativo, caracterizado por alterações na estrutura dos vasos sanguíneos, derivadas de citocinas pró-inflamatórias, além de interações entre fatores de crescimento, estímulos hemodinâmicos e espécies reativas de oxigênio. Compreende modificações no crescimento, morte e migração celular, e na síntese e degradação da matriz extracelular. ${ }^{17-19}$

Um fator importante a ser considerado nas alterações vasculares encontradas durante a SM é a dislipidemia, uma condição caracterizada pelo aumento de colesterol e triglicerídeos na circulação e redução dos níveis de HDL. ${ }^{20}$ Numerosos estudos estabeleceram que a dislipidemia leva a uma resposta inflamatória na vasculatura, refletida pela ativação das células endoteliais, recrutamento de leucócitos e produção de citocinas pró-inflamatórias. ${ }^{21,22}$ Camundongos hipercolesterolêmicos alimentados com dieta hiperlipídica mostram remodelamento da camada média da artéria femoral, associado ao recrutamento de macrófagos. ${ }^{23}$ Neste contexto, há correlação positiva entre os níveis de colesterol circulantes, inflamação crônica e remodelamento vascular.

Atorvastatina, inibidora da $\beta$-hidroxi- $\beta$-metil-glutaril coenzima A redutase, se apresenta como uma das estatinas mais eficazes no tratamento das dislipidemias, reduzindo a produção de colesterol LDL e aumentando a produção de colesterol HDL. ${ }^{24,25}$ Não obstante, nas últimas décadas, crescentes evidências, sejam experimentais ou clínicas, se acumularam para apoiar a ideia de que a atorvastatina exerce efeitos cardiovasculares benéficos, independentemente de seus efeitos primários. ${ }^{26} \mathrm{Em}$ um modelo experimental de SM, a atorvastatina foi capaz de melhorar a reatividade e reduzir o remodelamento estrutural de artérias de resistência; ambos os efeitos foram associados à redução da inflamação e do estresse oxidativo. ${ }^{27}$ Além disso, a atorvastatina é capaz de inibir a secreção de metaloproteinase de matriz-9 em células vasculares e suprimir a expressão do fator de transformação do crescimento beta, diminuindo a fibrose vascular. ${ }^{28-30}$ Esses efeitos in vitro da atorvastatina também estão associados à sua capacidade anti-inflamatória. ${ }^{31}$ No contexto clínico, a atorvastatina reduz processos ateroscleróticos ${ }^{32}$ e quadros clínicos de síndrome coronária aguda. ${ }^{33}$ De fato, dentre os diversos efeitos pleiotrópicos da atorvastatina, é robusta a sua capacidade vasoprotetora. Ainda assim, não há evidências suficientes que atestam sobre os efeitos e os possíveis mecanismos envolvidos nas ações da atorvastatina, sob a perspectiva do músculo liso vascular e, de forma inédita, sob o PVAT como tecidos inflamatórios em condições de SM. Assim, neste estudo, hipotetizamos que, durante a SM, ocorre remodelamento vascular a níveis de músculo liso vascular e PVAT, ocasionado pelo aumento da inflamação vascular. Além disso, o tratamento com atorvastatina pode ser capaz de reduzir a inflamação vascular e, consequentemente, reverter os prejuízos associados à SM.

\section{Métodos}

\section{Animais e delineamento experimental}

Todos os procedimentos experimentais foram analisados pela Comissão de Ética no Uso de Animais da Universidade Federal de Jataí (Protocolo 02/2019). Camundongos Swiss machos, de 3 semanas de idade, foram adquiridos do Biotério Central da Universidade Federal de Goiás - Regional Goiânia - e acondicionados no Biotério de Experimentação Animal da Universidade Federal de Jataí, com temperatura controlada de $22 \pm 2^{\circ} \mathrm{C}$, umidade de $50 \pm 5 \%$ e ciclos claro-escuro de 12 horas, com livre acesso a água e alimento. Os camundongos foram mantidos durante todo o período experimental em caixas de polipropileno (comprimento [30] $\times$ largura [20] $\times$ altura [13] cm), na proporção de 3 camundongos por caixa.

Após uma semana de aclimatização, os camundongos receberam dieta controle ou dieta hiperglicídica por 18 semanas. Após 14 semanas de dietas, os camundongos foram tratados (diariamente/turno vespertino) por gavagem com veículo (solução salina) ou atorvastatina [(20mg/kg), Sigma-Aldrich, \#PZ0001, Alemanha] durante 4 semanas. $^{34}$ 
A dieta controle constitui-se em 22\% de proteína, 70\% de carboidrato e $8 \%$ de gordura, enquanto a dieta hiperglicídica constitui-se em $10 \%$ de proteína, $80 \%$ de carboidrato e $10 \%$ de gordura. A dieta hiperglicídica foi formada por 33\% de dieta controle (Nuvilab® CR1, Nuvital, Brasil), 33\% de leite condensado e $7 \%$ de sacarose por peso da dieta controle, sendo o restante água. ${ }^{6} \mathrm{O}$ conteúdo energético foi de $12,16 \mathrm{~kJ} / \mathrm{g}$ para a dieta controle e $13,35 \mathrm{~kJ} / \mathrm{g}$ para a dieta hiperglicídica. Os camundongos foram eutanasiados por excesso de anestésicos (cetamina e xilazina, $140 \mathrm{mg} / \mathrm{kg}$ e $12 \mathrm{mg} / \mathrm{kg}$, respectivamente, via intraperitoneal).

\section{Perfil nutricional e murinométrico do modelo experimental}

O perfil nutricional foi determinado pelo consumo de alimentos e consequente ingestão calórica, além da eficiência alimentar. A ingestão calórica (por camundongo) foi calculada a partir da ingestão semanal de alimentos, multiplicada pelo valor energético da dieta $(\mathrm{g} \times \mathrm{kcal})$. Com a finalidade de analisar a capacidade de o camundongo converter a energia consumida em massa corporal, a eficiência alimentar foi calculada, dividindo-se o ganho total de massa corporal (g) pela energia total ingerida $(\mathrm{kcal})$, em porcentagem. O perfil murinométrico foi determinado pela análise da massa e gordura corporal. A massa corporal dos camundongos foi aferida semanalmente, utilizando balança analítica de precisão, e a obesidade foi caracterizada, ao final das dietas e tratamentos, pelo índice de adiposidade \{[gordura corporal (g)/peso corporal final (g) $\times 100\}$, sendo a gordura corporal calculada pela soma das gorduras epididimal, retroperitoneal e visceral. ${ }^{35}$

\section{Obtenção de soro e PVAT}

Os camundongos foram submetidos à privação alimentar por 8 horas. Após eutanásia, amostras de sangue foram coletadas por punção cardíaca e transferidas para tubos secos. O sangue foi centrifugado a 2.500 rpm por 15 minutos para separação do soro, utilizado nas dosagens bioquímicas e citocinas. O PVAT foi retirado mecanicamente dos segmentos de aorta torácica, congelado em nitrogênio líquido, pulverizado e homogeneizado em tampão fosfato salina (PBS) gelado. O extrato tecidual foi centrifugado a $13.000 \mathrm{rpm}$ por 20 minutos para separação do material insolúvel. O sobrenadante foi coletado para as dosagens de citocinas.

\section{Avaliação do perfil lipídico e glicêmico}

Foram determinados os níveis séricos de colesterol total, triglicerídeos e HDL (lipoproteínas de alta densidade), por métodos enzimáticos (kits Labtest, Brasil), em amostras de soros provenientes da centrifugação do sangue total. A partir da concentração sérica de triglicerídeos, foram calculadas as concentrações de VLDL (lipoproteínas de muita baixa densidade), utilizando-se o seguinte cálculo: VLDL $(\mathrm{mg} / \mathrm{dL})=$ triglicerídeos $(\mathrm{mg} / \mathrm{dL}) / 5$. A partir da concentração de HDL e VLDL, foi calculada a concentração de LDL (lipoproteínas de baixa densidade), utilizando o cálculo: LDL $(\mathrm{mg} / \mathrm{dL})=$ colesterol total - HDL - VLDL. ${ }^{36}$ A glicemia foi determinada após o tratamento com dietas. Uma gota de sangue foi colocada em fitas individuais específicas para leitura em glicosímetro (Accu-Check Active, Alemanha).

\section{Teste oral de tolerância à glicose (TOTG)}

Um dia anterior ao final da $18^{\underline{a}}$ semana de dietas e tratamentos, após 8 horas de jejum, a glicemia de cada camundongo foi verificada por glicosímetro (Accu-Check Active, Alemanha), caracterizando a glicemia do tempo zero. Em seguida, glicose na dose de $2 \mathrm{~g} / \mathrm{kg}$ foi administrada aos camundongos por gavagem. A partir desse momento, o cronômetro foi acionado e novas determinações da glicemia foram feitas nos tempos de 30, 60, 90 e 120 minutos. $^{37}$

\section{Avaliação morfoestrutural do músculo liso vascular e do PVAT}

Aortas da região torácica foram removidas e então fixadas por 24 horas em paraformaldeído a 4\%. As etapas seguintes envolveram a desidratação em concentrações crescentes de álcool etílico (70\%, 80\%, 90\% e 100\%) por 120 minutos, com posterior diafanização em álcool etílico absoluto cada (1:1) 1, 2 e 3 por 120 minutos e em xilol 1, 2 e 3 por 30 minutos, com posterior inclusão em parafina. As aortas foram incluídas em parafina e cortadas em micrótomo na espessura de 4,5 $\mu \mathrm{m}$, para posterior coloração.

Para a coloração de hematoxilina e eosina, os cortes em lâminas foram submetidos ao processo de desparafinização, sendo mantidos em estufa a $65^{\circ} \mathrm{C}$, , posterior imersão em xilol 1 e 2 por 20 minutos. Em seguida, foram imergidos em soluções decrescentes $(100 \%, 90 \%, 70 \%$ e 50\%) de álcool etílico durante 5 minutos e imersos em água destilada por 10 minutos. Seguida a hidratação, as lâminas foram imersas em corante hematoxilina por 6 minutos e, logo após, lavadas em água corrente por 10 minutos. A seguir, foram imersas em corante eosina por 6 minutos. Os cortes corados foram novamente desidratados e diafanizados, sendo montados com meio de montagem Permount ${ }^{\circledR}$ (Fisher Scientific, EUA) e lamínulas.

Imagens coloridas foram obtidas por câmera digital acoplada à microscópio óptico e analisadas pelo programa ImageJ (Instituto Nacional de Saúde, EUA). Para análise das imagens, foi delimitado o lúmen, a túnica média e adventícia com o objetivo de calcular as seguintes variáveis morfométricas: área de secção transversal (AST) da túnica média e espessura da túnica média. O volume dos adipócitos (v) foi calculado a partir da fórmula $v=\pi \mathrm{d}^{3} / 6$, em que (d) representa o diâmetro dos adipócitos. A massa do adipócito se deu pelo volume multiplicado pela densidade $\left(0,92 \mathrm{~g} / \mathrm{cm}^{3}\right)$. O número de adipócitos foi determinado dividindo-se a massa do PVAT pela massa média de adipócitos. ${ }^{38}$

\section{Determinação dos níveis de citocinas inflamatórias}

Os níveis de TNF- $\alpha$ e IL- 6 no soro e no PVAT foram feitos pelo método imunoenzimático (ELISA) utilizando kits DuoSet ELISA Development Systems (R\&D Systems, EUA) de acordo com as informações do fabricante. Placas de microtitulação (96 poços) foram recobertas com $50 \mu \mathrm{L} /$ poço dos anticorpos específicos anti-TNF- $\alpha$ e anti-IL-6, nas concentrações descritas pelo fabricante, diluídos em PBS e incubados overnight a 4으. As placas foram lavadas com PBS/Tween-20 (0,05\%), e as ligações não específicas foram bloqueadas com $100 \mu \mathrm{L}$ de PBS contendo albumina do soro bovino 1\% durante 2 horas 
em temperatura ambiente. Posteriormente, as amostras foram adicionadas, seguindo nova incubação por 2 horas em temperatura ambiente. Após esse período, as placas foram lavadas e foram adicionados $50 \mu \mathrm{L}$ dos anticorpos biotinilados específicos para cada citocina. Após 2 horas, as placas foram lavadas e o conjugado estreptavidina-peroxidase, na diluição de 1:40, foi adicionado a cada poço. As placas foram incubadas por 1 hora em temperatura ambiente. Posteriormente, as placas foram lavadas e adicionou-se a dosagem de $100 \mu \mathrm{L}$ do substrato tetrametilbenzidina. A densidade ótica foi medida a $630 \mathrm{~nm}$ no espectrofotômetro SpectraMAX 190 Microplate Reader (Molecular Devices, EUA). Os níveis de citocinas contidas nas amostras foram calculados a partir de uma curva padrão com 11 pontos obtidos por diluição seriada. Os resultados foram expressos em $\mathrm{pg} / \mathrm{mL}$ para amostras de soro, e pg/mg de proteína para amostras de PVAT.

\section{Análise estatística}

Os resultados foram expressos como média \pm desvio padrão (DP). Os dados seguiram distribuição normal de acordo com o teste de Kolmogorov-Smirnov. Os resultados foram analisados pelo teste de análise de variância de duas vias (Two-Way ANOVA), seguido do pós-teste Tukey. O nível de significância mínima aceitável foi $p<0,05$. Por conveniência, o número de camundongos para cada grupo experimental foi igual a 6. O programa Prisma, versão 8.0 (GraphPad Software Inc., EUA) foi utilizado para analisar esses parâmetros, bem como para a construção dos gráficos.

\section{Resultados}

\section{Perfil nutricional e murinométrico}

A ingestão alimentar e a consequente ingestão calórica foram maiores nos camundongos mantidos com dieta hiperglicídica, bem como a eficiência alimentar; no entanto, este último parâmetro foi reduzido após o tratamento com atorvastatina. Os parâmetros murinométricos, como ganho de massa, depósitos de gorduras epididimal, retroperitoneal e visceral foram maiores nos camundongos mantidos em dieta hiperglicídica quando comparados aos camundongos mantidos em dieta controle. O tratamento com atorvastatina promoveu redução de tais parâmetros (Tabela 1). Os camundongos mantidos em dieta hiperglicídica apresentaram maior aumento de massa corporal quando comparados aos camundongos mantidos em dieta controle. Além disso, camundongos mantidos em dieta hiperglicídica e tratados com atorvastatina apresentaram redução de massa corporal (Figura 1A). O índice de adiposidade, parâmetro para determinar a obesidade, foi maior nos camundongos mantidos em dieta hiperglicídica quando comparados aos camundongos mantidos em dieta controle. O tratamento com atorvastatina promoveu redução no índice de adiposidade dos camundongos mantidos em dieta hiperglicídica (Figura 1B).

\section{Perfil lipídico}

Os camundongos mantidos em dieta hiperglicídica apresentaram dislipidemia quando comparados aos camundongos que receberam dieta controle. A dislipidemia pode ser observada a partir do aumento nos níveis de colesterol total (Figura 2A), em que as frações LDL (Figura 2B) e VLDL (Figura 2C) se encontraram aumentadas e a fração HDL (Figura 2D), diminuída. Houve também aumento nos níveis de triglicerídeos dos camundongos que receberam dieta hiperglicídica em comparação aos que foram mantidos em dieta controle (Figura 2E). Os camundongos mantidos em dieta hiperglicídica e que foram tratados com atorvastatina mostraram redução de tais parâmetros lipídicos.

\section{Perfil glicêmico}

A glicemia de jejum dos camundongos mantidos em dieta hiperglicídica estava aumentada quando comparada à glicemia de jejum dos camundongos mantidos em dieta controle. Os camundongos que receberam dieta hiperglicídica e tratamento com atorvastatina não mostraram redução na concentração glicêmica (Figura 3A). No TOTG (Figura 3B), camundongos que receberam dieta hiperglicídica apresentaram aumento na concentração glicêmica quando comparados aos camundongos que receberam dieta controle, sendo que, mesmo com o passar do tempo, os níveis de glicose não retornaram aos níveis basais, como observado nos camundongos que receberam dieta controle. Além disso, camundongos que receberam dieta hiperglicídica e tratados

Tabela 1 - Comportamento alimentar e avaliação ponderal dos depósitos de gordura corporal

\begin{tabular}{|c|c|c|c|c|}
\hline & Dieta C + Veículo & Dieta C + Atorvastatina & Dieta H + Veículo & Dieta H + Atorvastatina \\
\hline Ingestão alimentar (g/semana) & $22,6 \pm 0,3$ & $21,3 \pm 0,4$ & $31,7 \pm 0,2$ & $31,1 \pm 0,5$ \\
\hline Ingestão calórica (kcal/semana) & $274,8 \pm 3,7$ & $259,1 \pm 4,9$ & $423,1 \pm 2,67^{*}$ & $415,2 \pm 6,7$ \\
\hline Eficiência alimentar (\%) & $0,51 \pm 0,01$ & $0,53 \pm 0,01$ & $0,64 \pm 0,02^{*}$ & $0,47 \pm 0,03^{\#}$ \\
\hline Ganho de massa (g) & $25,0 \pm 2,31$ & $24,5 \pm 2,25$ & $37,3 \pm 1,52^{*}$ & $27,9 \pm 1,22^{\#}$ \\
\hline Gordura epididimal (g) & $0,97 \pm 0,12$ & $1,13 \pm 0,11$ & $2,68 \pm 0,24^{*}$ & $1,31 \pm 0,09^{\#}$ \\
\hline Gordura retroperitoneal (g) & $0,32 \pm 0,06$ & $0,61 \pm 0,19$ & $1,88 \pm 0,12^{*}$ & $0,93 \pm 0,19^{\#}$ \\
\hline Gordura visceral (g) & $0,75 \pm 0,15$ & $0,94 \pm 0,13$ & $1,67 \pm 0,11^{*}$ & $1,01 \pm 0,17^{\#}$ \\
\hline
\end{tabular}

Dados expressos como média $\pm D P$. *, $p<0,05$ vs. dieta $C+$ veículo; *, $p<0,05$ vs. dieta $H+$ veículo ( $n=6$ para cada grupo experimental). Dieta C: dieta controle, dieta $\mathrm{H}$ : dieta hiperglicídica. 
A

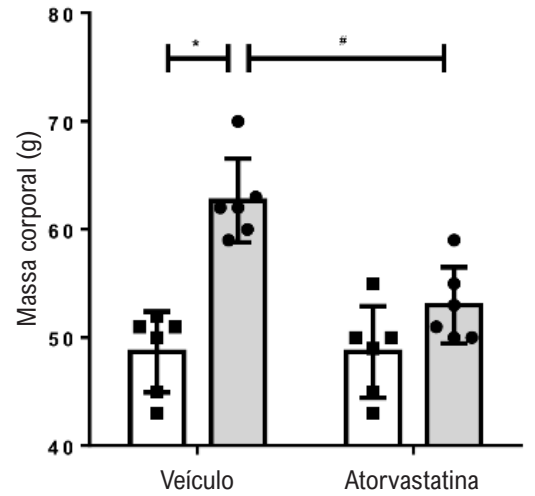

B

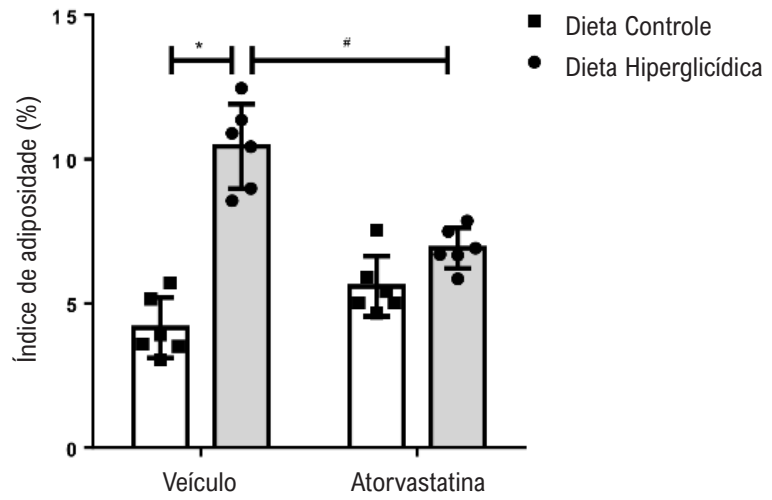

Figura 1 - Atorvastatina atenua a obesidade associada à síndrome metabólica induzida por dieta hiperglicídica. As figuras mostram valores de massa corporal (A) e índice de adiposidade (B) de camundongos mantidos por 18 semanas em dieta controle ou dieta hiperglicídica, tratados com veículo ou atorvastatina (20 mg/kg por 4 semanas). Os resultados representam a média $\pm D P$. *, $p<0,05$ vs. dieta controle_veículo; \#, $p<0,05$ vs. dieta hiperglicídica_veículo ( $n=6$ para cada grupo experimental).

A

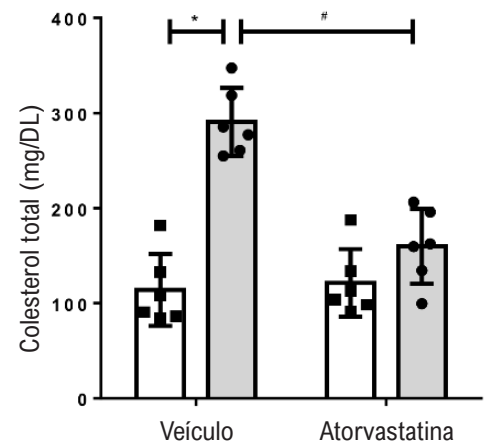

D

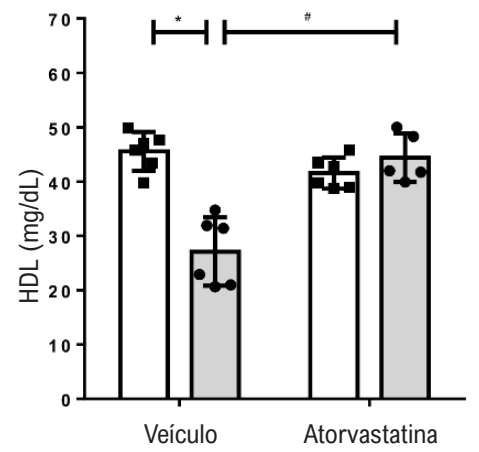

B

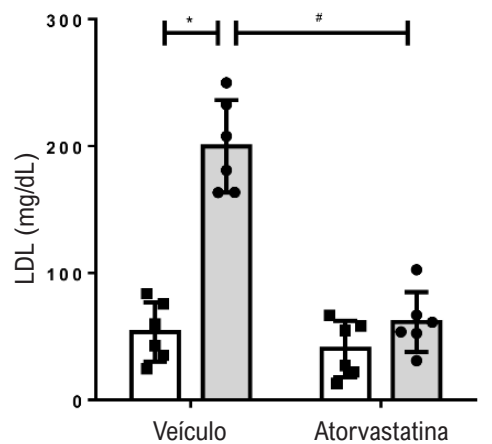

E

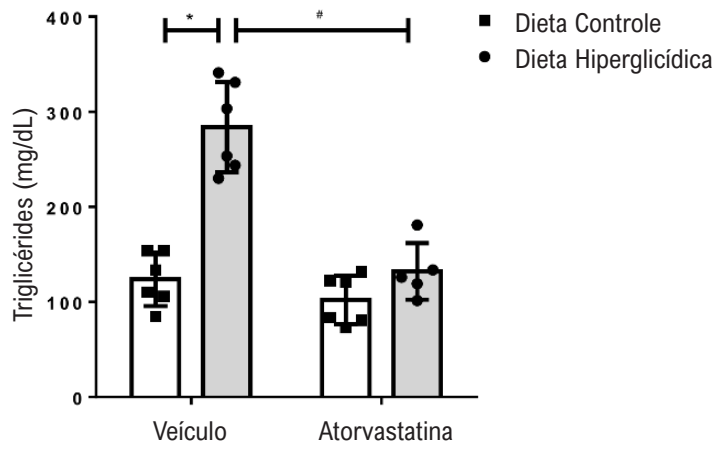

Figura 2 - Atorvastatina atenua a dislipidemia associada à síndrome metabólica induzida por dieta hiperglicídica. As figuras mostram niveis de colesterol total (A), LDL (B), VLDL (C), HDL (D) e niveis de triglicerídeos (E) de camundongos mantidos por 18 semanas em dieta controle ou dieta hiperglicídica, tratados com veículo ou atorvastatina (20 mg/kg por 4 semanas). Os resultados representam a média $\pm D P$. ${ }^{*}, p<0,05$ vs. dieta controle_veículo; $\#, p<0,05$ vs. dieta hiperglicídica_veículo ( $n=6$ para cada grupo experimental). 
com atorvastatina apresentaram, com o passar do tempo, menor glicemia. A área sob a curva reflete os efeitos citados (Figura 3C).

\section{Morfologia muscular lisa}

Camundongos mantidos em dieta hiperglicídica apresentaram aumento na AST vascular quando comparados aos camundongos mantidos em dieta controle. Camundongos que receberam dieta hiperglicídica e foram tratados com atorvastatina mostraram redução na AST (Figura 4A). De modo similar, camundongos que receberam dieta hiperglicídica apresentaram aumento na espessura da camada média do vaso quando comparados aos camundongos que receberam dieta controle. O tratamento com atorvastatina foi capaz de reduzir este aumento na espessura da camada média (Figura 4B).

\section{Morfologia do tecido adiposo perivascular}

Para verificar se houve alterações na morfologia do PVAT, foi avaliada a túnica adventícia a partir da medida e do número dos adipócitos presentes na mesma, sendo possível observar que os camundongos que receberam dieta hiperglicídica apresentaram adipócitos maiores (Figura 5A) e em menor número (Figura 5B) quando comparados com os camundongos que receberam dieta controle. O grupo que recebeu dieta hiperglicídica e tratamento com atorvastatina obteve redução no tamanho e aumento no número dos adipócitos.

\section{Perfil inflamatório}

Foram determinadas as concentrações de citocinas pró-inflamatórias TNF- $\alpha$ (Figuras 6A e 6C) e IL-6 (Figuras 6B e 6D) no soro e no PVAT. Os camundongos que receberam dieta hiperglicídica apresentaram maiores concentrações de TNF- $\alpha$ e IL-6, tanto no soro quanto no PVAT, quando comparados aos camundongos que receberam dieta controle. O tratamento com atorvastatina reduziu os níveis de TNF- $\alpha$ e IL-6, tanto no soro quanto no PVAT, de camundongos mantidos em dieta hiperglicídica.

\section{Discussão}

A dieta hiperglicídica resultou em mudanças murinométricas e metabólicas em camundongos. Silva et al. ${ }^{39}$ submeteu um modelo experimental à dieta semelhante, verificando aumentos em idênticos parâmetros utilizados neste estudo. ${ }^{39}$ Neste contexto, Leopoldo et al., ${ }^{40}$ utilizando modelos experimentais, definiu as categorias de adiposidade após

A

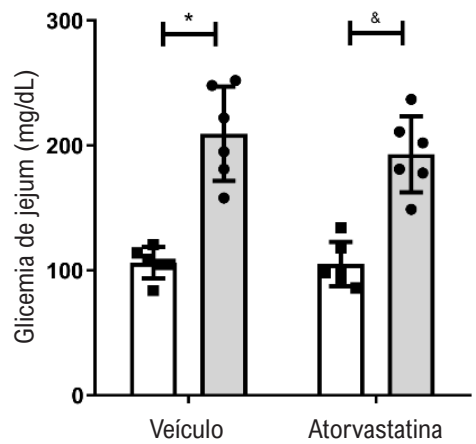

B

C

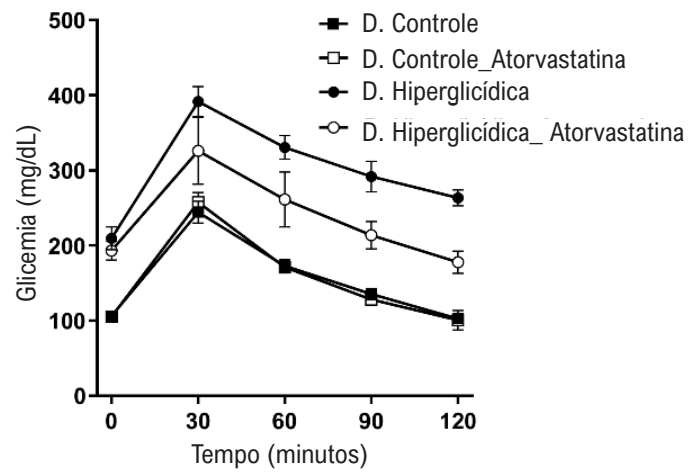

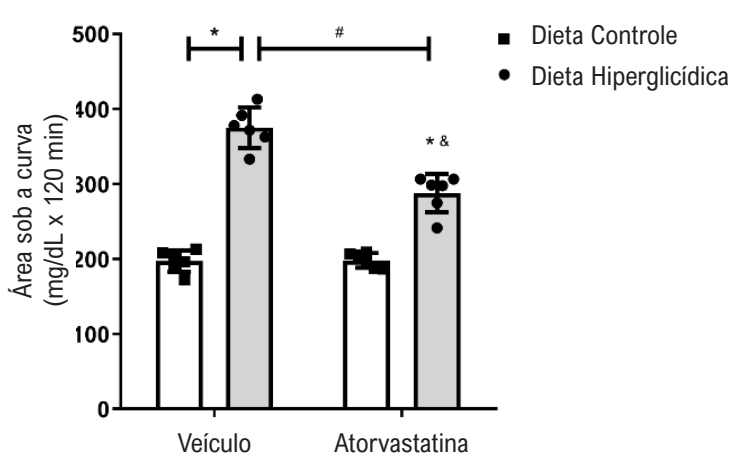

Figura 3 - Atorvastatina atenua a intolerância à glicose associada à síndrome metabólica induzida por dieta hiperglicídica. As figuras mostram níveis de glicemia em jejum (A), curvas glicêmicas (B) e área sob a curva (C), obtidas no teste oral de tolerância à glicose de camundongos mantidos por 18 semanas em dieta controle ou dieta hiperglicídica, tratados com veículo ou atorvastatina (20 mg/kg por 4 semanas). Os resultados representam a média $\pm D P$. *, $p<0,05 \mathrm{vs}$. dieta controle_veículo; $\&, p<0,05$ vs. dieta controle_atorvastatina; \#, $p<0,05$ vs. dieta hiperglicídica_veículo ( $n=6$ para cada grupo experimental). 


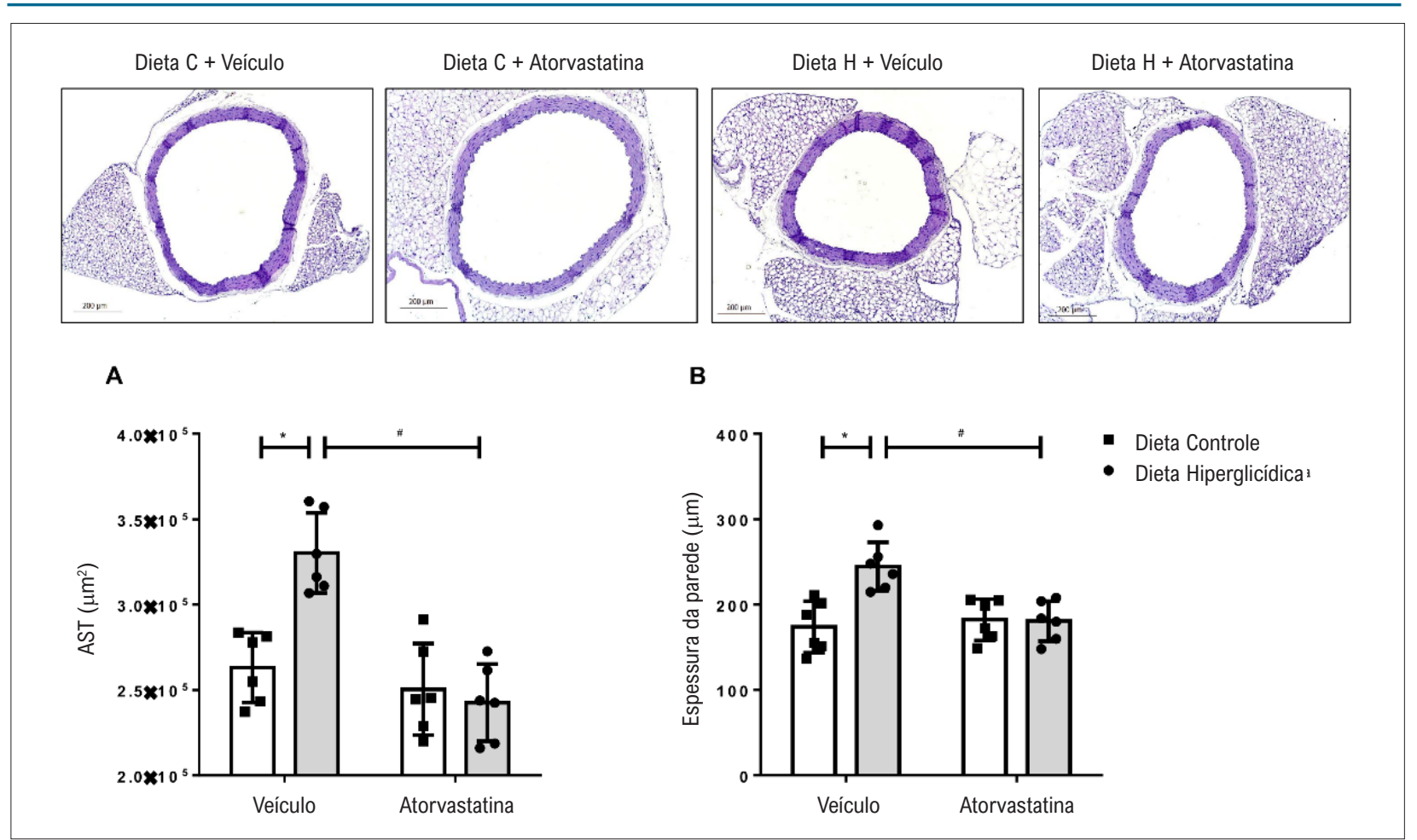

Figura 4 - Atorvastatina atenua o remodelamento do músculo liso vascular associado à síndrome metabólica induzida por dieta hiperglicídica. As figuras mostram imagens representativas, tamanho da AST (A) e espessura da parede do vaso (B) de camundongos mantidos por 18 semanas em dieta controle ou dieta hiperglicídica, tratados com veículo ou atorvastatina (20 mg/kg por 4 semanas). Os resultados representam a média $\pm D P$. ${ }^{*}, p<0,05 \mathrm{vs}$. dieta controle_veículo; ", $p<0,05$ vs. dieta hiperglicídica_veículo ( $n=6$ para cada grupo experimental). Dieta C: dieta controle, Dieta H: dieta hiperglicídica.

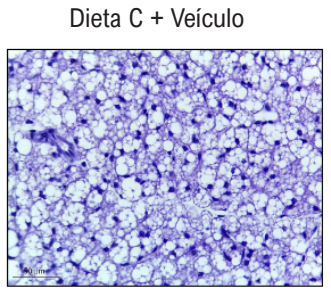

A

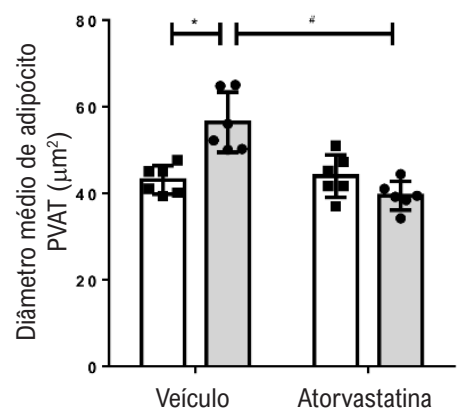

Dieta C + Atorvastatina

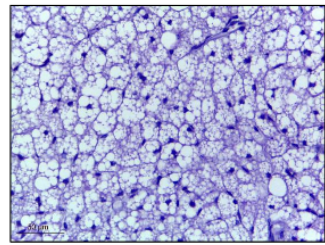

Dieta $\mathrm{H}+$ Veículo

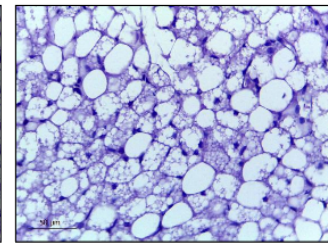

Dieta $\mathrm{H}+$ Atorvastatina

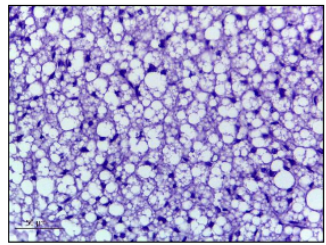

B

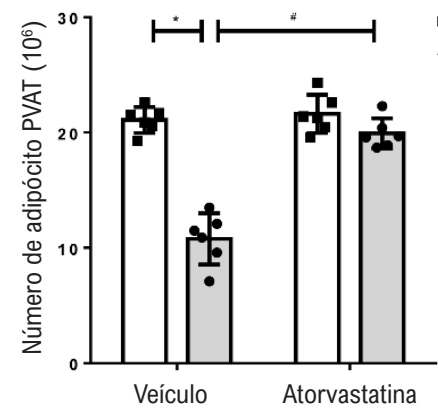

Figura 5 - Atorvastatina atenua o remodelamento do PVAT associado à síndrome metabólica induzida por dieta hiperglicídica. As figuras mostram imagens representativas $(A)$ e o tamanho de adipócitos PVAT $(B)$ de camundongos mantidos por 18 semanas em dieta controle ou dieta hiperglicídica, tratados com veículo ou atorvastatina (20 mg/kg por 4 semanas). Os resultados representam a média $\pm D P$. *, $p<0,05$ vs. dieta controle_veículo; *, $p<0,05$ vs. dieta hiperglicídica_veículo ( $n=6$ para cada grupo experimental). Dieta C: dieta controle, Dieta H: dieta hiperglicídica. 


\section{Artigo Original}

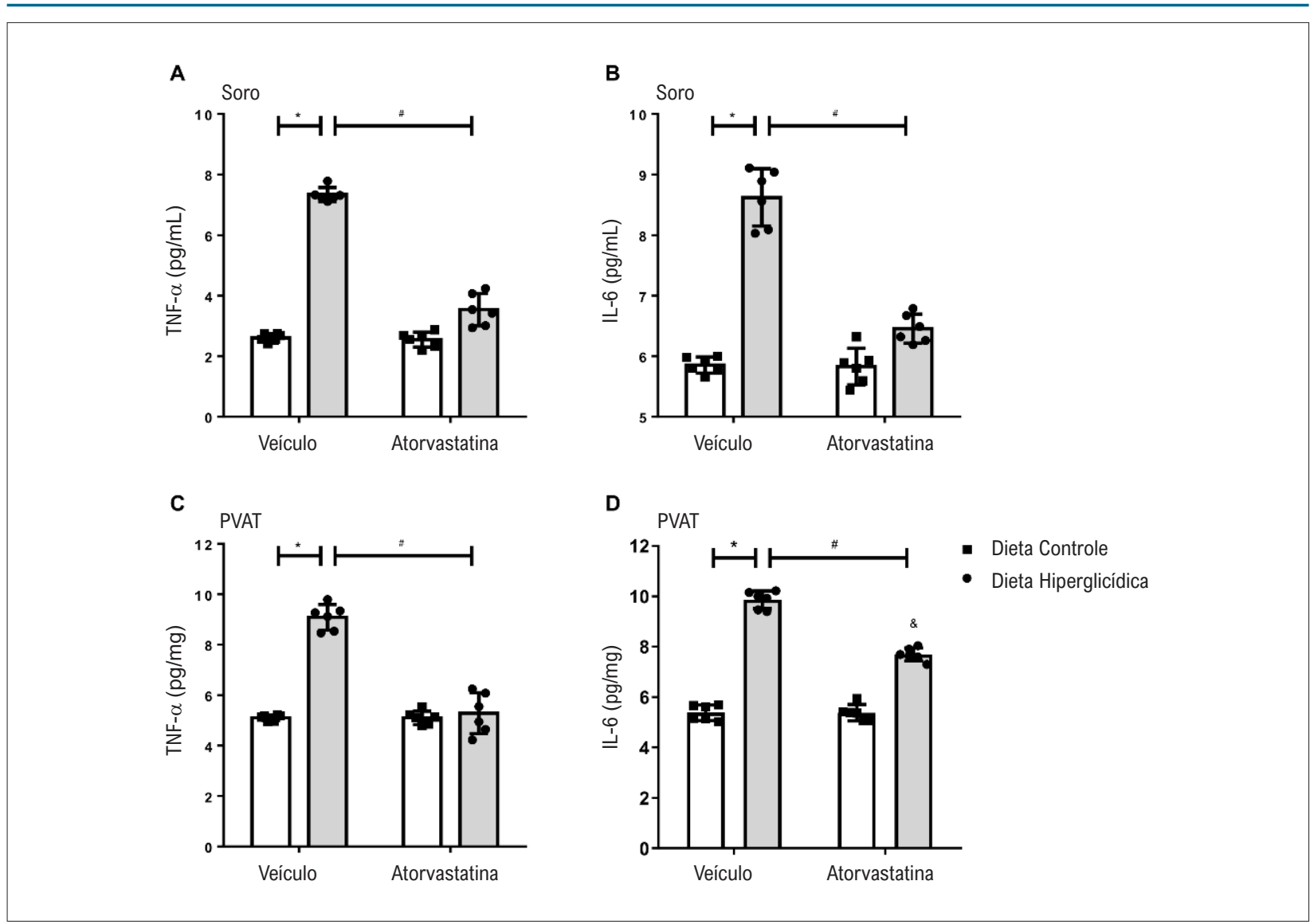

Figura 6 - Atorvastatina atenua os aumentos nas concentrações de citocinas pró-inflamatórias associados à síndrome metabólica induzida por dieta hiperglicídica. As figuras mostram níveis de TNF-a (A e C) e IL-6 (B e D) no soro e no PVAT, respectivamente, de camundongos mantidos por 18 semanas em dieta controle ou dieta hiperglicídica, tratados com veículo ou atorvastatina (20 mg/kg por 4 semanas). Os resultados representam a média $\pm D P$. * $, p<0,05$ vs. dieta controle_veículo; \&, $p<0,05$ vs. dieta controle_atorvastatina; \#, $p<0,05$ vs. dieta hiperglicídica_veículo ( $n=6$ para cada grupo experimental).

submissão à dieta hiperglicídica, tais quais variam entre: normal - índice de adiposidade (\%) 4,17 a 4,55; excesso de peso - índice de adiposidade (\%) 5,69 a 6,23; e obesidade índice de adiposidade (\%) 7,53 a 10,02. ${ }^{40}$ Nossos resultados demostram índice de adiposidade de aproximadamente 10\% para os camundongos mantidos em dieta hiperglicídica, o que classifica o modelo experimental como obeso.

A glicose é um carboidrato que atua como uma das principais fontes de energia para o organismo e, em excesso, através de vias metabólicas, é armazenada em forma de glicogênio. Se a capacidade de armazenamento da ingesta de carboidratos excede a glicogênese, o excedente é convertido em triacilgliceróis e é incorporado a colesteróis provenientes da dieta e a apolipoproteínas, responsáveis pelo transporte de triglicerídeos até os tecidos de armazenamento, como, por exemplo, o tecido adiposo. ${ }^{41}$ Dessa forma, o excesso de carboidratos ofertados na dieta dos camundongos corrobora para o aumento da massa corporal e do índice de adiposidade.

Os camundongos mantidos em dieta hiperglicídica apresentaram dislipidemia e aumento da glicose sérica em jejum. Mullugeta et al., ${ }^{42}$ demostrou, em portadores de diabetes melito do tipo 2, que a hiperglicemia e a resistência à insulina estão associados à dislipidemia, ou seja, hiperglicemia promove prejuízos no metabolismo lipídico. Além disso, demostrou-se que indivíduos hiperglicêmicos apresentam valores séricos de triglicerídeos, colesterol total e frações aumentados quando comparados aos indivíduos normoglicêmicos. ${ }^{42,43}$

O tratamento com atorvastatina se mostrou eficiente para o controle dos níveis de colesterol total e frações. Além disso, a atorvastatina reduziu os níveis de triglicerídeos, a massa corporal e o índice de adiposidade. Silva et al. ${ }^{44}$ também demostrou a eficácia da atorvastatina em doses diárias de 10 mg ou 20 mg sobre a correção do perfil lipídico de indivíduos, em que, além da terapêutica sobre o colesterol, os triglicerídeos também foram reduzidos. ${ }^{44}$ Sabe-se que VLDL e LDL são lipoproteínas constituídas por quantidades significativas de triacilgliceróis; sendo assim, propomos que, com a redução dos níveis de VLDL e LDL, por meio da ação da atorvastatina, é observada diminuição de triglicerídeos. Parhofer et al. ${ }^{45}$ descreve o efeito redutor de triglicerídeos principalmente pela a inibição da síntese de colesterol, e também salientam que VLDL e LDL têm o mesmo mecanismo de remoção. Assim, ao reduzir a quantidade de 
LDL na circulação sanguínea, pode-se também diminuir significantemente os níveis de VLDL. ${ }^{45}$

A dieta hiperglicídica, de acordo com o TOTG, induziu nos camundongos intolerância à glicose. Sabe-se que a exposição crônica do organismo à hiperglicemia acaba afetando a atividade insulínica no que diz respeito a síntese, secreção e ação deste hormônio, o que, de fato, é observado no diabetes melito tipo $2 .{ }^{6}$ Sendo assim, é possível sugerir que os resultados levantados no perfil glicêmico de camundongos mantidos em dieta hiperglicídica corroboram para o desenvolvimento de diabetes melito tipo 2. Kissebah et al., ${ }^{46}$ demonstrou que o aumento da gordura visceral está associado à intolerância à glicose e à resistência à insulina; Taylor et al., ${ }^{47}$ demonstrou que a gordura visceral está relacionada com o desenvolvimento de diabetes melito. ${ }^{46,47}$ Estudos descrevem que o aumento da gordura visceral predispõe a produção e secreção de citocinas, tais como IL-6 e TNF- $\alpha$, e adipocinas como resistina e adiponectina, que estão associadas ao desenvolvimento de resistência à insulina e disfunções no metabolismo e no controle da glicose, por interferência na sinalização intracelular na insulina. ${ }^{48,49}$ O aumento da gordura visceral, bem como o aumento de TNF- $\alpha$ e IL-6, justificam o desequilíbrio no perfil glicêmico.

A terapia com atorvastatina foi capaz de atenuar a intolerância à glicose nos camundongos mantidos em dieta hiperglicídica. Huptas, et al. ${ }^{50}$ obteve resultados promissores em relação ao tratamento de indivíduos portadores de SM com atorvastatina, ele demostrou melhoras no metabolismo da glicose mediante a redução da intolerância à glicose e da resistência à insulina. Suzuki et al., ${ }^{51}$ utilizando modelos experimentais de intolerância à glicose e de resistência à insulina, realizou TOTG e teste de tolerância à insulina (TTI) com ou sem o tratamento com atorvastatina, e demostrou que o grupo tratado tem TOTG e TTI reduzidos em comparação ao grupo que recebeu veículo. ${ }^{50,51}$

Não se sabe ao certo por quais mecanismos a atorvastatina melhora os parâmetros relacionados ao metabolismo da glicose. Tal melhora pode estar relacionada à diminuição do processo de gliconeogênese. ${ }^{51}$ No entanto, é possível sugerir que tal redução da intolerância à glicose pode estar relacionada com a redução dos depósitos de gordura visceral; além disso, pode estar relacionada à redução da produção de citocinas (TNF- $\alpha$ e IL-6), que são potenciais causas de disfunções metabólicas.

De acordo com Mulvany (1999), o remodelamento vascular pode ser classificado a partir de medidas da AST e espessura da parede. O aumento da AST e da espessura da parede é característico de remodelamento do tipo hipertrófico. ${ }^{52}$ Uma das causas do remodelamento vascular é a inflamação mediada por aumento de citocinas pró-inflamatórias. Estudos relacionados à SM mostram que a hipertrofia do PVAT resulta na migração, ativação de células imunes e aumento da secreção dessas citocinas, levando a um processo inflamatório crônico de baixa intensidade. ${ }^{53-55}$

As mudanças na estrutura e na composição do PVAT refletem-se na túnica média do vaso, pois, fisiologicamente, o PVAT é constituído de adipócitos que agem como reguladores da proliferação de células da musculatura lisa vascular (CMLV). A mudança por adipócitos não especializados culmina em aumento da proliferação de CMLV e hipertrofia da musculatura lisa. Esta hipertrofia apresenta papel significativo em doenças vasculares como, por exemplo, reestenose e hipertensão arterial. ${ }^{56}$

TNF- $\alpha$ e IL-6 se encontram em concentrações aumentadas durante a inflamação crônica presente na obesidade, na resistência à insulina e na SM. Além disso, há clara relação do aumento dessas citocinas em mudanças fenotípicas do PVAT. ${ }^{57,58}$ Citocinas pró-inflamatórias promovem o remodelamento e a disfunção vascular, uma vez que concentrações aumentadas de TNF- $\alpha$ promovem a hiperplasia neointimal e a disfunção endotelial. De forma semelhante, a IL-6, quando em concentrações aumentadas, pode induzir a infiltração de macrófagos no PVAT, contribuindo para o desenvolvimento de aneurisma e remodelamento vascular associado à inflamação do PVAT. ${ }^{59,60}$

A partir de uma dieta hiperlipídica, foi induzida em coelhos hipercolesterolemia para levantamento das alterações que a condição poderia desenvolver nas artérias pulmonares dos animais. As artérias apresentaram aumento da proliferação de CMLV, bem como hipertrofia da túnica média e hiperplasia da túnica íntima, além disso, no tecido pulmonar, havia grande infiltrado de células inflamatórias como, por exemplo, macrófagos; houve aumento de IL-6 no soro desses animais. Os coelhos foram tratados com atorvastatina, evidenciando-se a reversão da condição hipercolesterolêmica, do remodelamento vascular e dos processos inflamatórios no tecido pulmonar. ${ }^{61}$ Similarmente, atorvastatina inibe o remodelamento vascular induzido por aldosterona, via redução de citocinas pró-inflamatórias. ${ }^{31}$

Como limitação desse estudo, não é possível estabelecer uma relação casuística direta entre o tratamento farmacológico com atorvastatina, as amenidades no perfil metabólico e o remodelamento vascular. A partir do delineamento experimental utilizado nesse estudo, mostramos que a atorvastatina reduz a adiposidade e melhora os perfis lipídicos, glicêmicos e inflamatórios. Na literatura, é clássico que tais fatos são benéficos para a estrutura e a função vascular. Sendo assim, não temos a clareza absoluta de que o remodelamento vascular atenuado se deu por ações diretas da atorvastatina sobre a vasculatura, ou por meio de efeitos adjacentes ao metabolismo. Ressaltamos que, em organismos multicomplexos, mecanismos isolados não justificam a gênese de doenças, sendo que, nesse contexto, há sempre uma vasta interação entre mecanismos e sistemas para tal. Portanto, independentemente da ação direta ou pleiotrópica da atorvastatina, a terapia final se mostra promissora para o contexto deste estudo.

\section{Conclusão}

Em resumo, este estudo mostra que o tratamento com atorvastatina atenua os danos metabólicos associados à síndrome metabólica induzida por dieta hiperglicídica, além de atenuar o remodelamento do músculo liso vascular e do PVAT, sendo esses efeitos associados à redução de citocinas pró-inflamatórias TNF- $\alpha$ e IL-6.

\section{Contribuição dos autores}

Concepção e desenho da pesquisa, Revisão crítica do manuscrito quanto ao conteúdo intelectual importante, Análise estatística e Redação do manuscrito: Carvalho KFS, Costa RM; Obtenção de dados: Carvalho KFS, Ferreira AAM, 
Barbosa NC, Alves JV; Análise e interpretação dos dados: Carvalho KFS, Ferreira AAM, Barbosa NC, Alves JV, Costa RM; Obtenção de financiamento: Costa RM.

\section{Potencial conflito de interesse}

Não há conflito com o presente artigo

\section{Referências}

1. Ribeiro ALP, Duncan BB, Brant LCC, Lotufo PA, Mill JG, Barreto SM. Cardiovascular health in Brazil. Circulation. 2016; 133(4):422-33.

2. Lotufo PA. Cardiovascular and cancer mortality in Brazil from 1990 to 2017. Sao Paulo Med J. 2019; 137(2):107-111.

3. Punthakee Z, Goldenberg R, Katz P. Definition, classification and diagnosis of diabetes, prediabetes and metabolic syndrome. Can J Diabetes. 2018; 42(1):S10-S15.

4. Lam DW, LeRoith D. Metabolic Syndrome. [Updated 2019 Feb 11]. In: Feingold KR, Anawalt B, Boyce A, et al., editors. Endotext. South Dartmouth (MA): MDText.com, Inc.; 2000.

5. Silva MA, Cau SB, Lopes RA, Manzato CP, Neves KB, Bruder-Nascimento T, et al. Mineralocorticoid receptor blockade prevents vascular remodelling in a rodent model of type 2 diabetes mellitus. Clin Sci. 2015; 129(7):533-45.

6. Costa RM, Silva JF, Alves JV, Dias TB, Rassi DM, Garcia LV, et al. Increased O-GlcNAcylation of endothelial nitric oxide synthase compromises the anticontractile properties of perivascular adipose tissue in metabolic syndrome. Front Physiol. 2018; 9(eCollection 2018):341.

7. Tran V, Silva TM, Sobey CG, Lim K, Drummond GR, Vinh A, et al. The vascular consequences of metabolic syndrome: rodent models, endothelial dysfunction, and current therapies. Front Pharmacol. 2020; 11(eCollection 2020):148.

8. Virdis A. Endothelial dysfunction in obesity: role of inflammation. High Blood Press Cardiovasc Prev. 2016; 23(2):83-85.

9. Xia N, Li H. The role of perivascular adipose tissue in obesity-induced vascular dysfunction. Br J Pharmacol. 2017; 174(20):3425-42.

10. Costa RM, Neves KB, Tostes RC, Lobato NS. Perivascular adipose tissue as a relevant fat depot for cardiovascular risk in obesity. Front Physiol. 2018; 9(eCollection 2018):253.

11. Victorio JA, da Costa RM, Tostes RC, Davel AP. Modulation of vascular function by perivascular adipose tissue: sex differences. Curr Pharm Des. $2020 ; 26(1): 1-12$.

12. Nosalski R, Guzik TJ. Perivascular adipose tissue inflammation in vascular disease. Br J Pharmacol. 2017; 174(20):3496-513.

13. Chatterjee TK, Stoll LL, Denning GM, Harrelson A, Blomkalns AL, Idelman $\mathrm{G}$, et al. Proinflammatory phenotype of perivascular adipocytes: influence of high-fat feeding. Circ Res. 2009; 104(4):541-9.

14. Costa RM, Fais RS, Dechandt CRP, Louzada-Junior P, Alberici LC, Lobato NS, et al. Increased mitochondrial ROS generation mediates the loss of the anti-contractile effects of perivascular adipose tissue in high-fat diet obese mice. Br J Pharmacol. 2017; 174(20):3527-41.

15. Anzai T. Inflammatory mechanisms of cardiovascular remodeling. Circ J. 2018; 82(3):629-35.

16. Ilatovskaya DV, Halade GV, DeLeon-Pennell KY. Adaptive immunity-driven inflammation and cardiovascular disease. Am J Physiol Heart Circ Physiol. 2019; 317(6):H1254-H1257.

17. Aroor AR, Jia G, Sowers JR. Cellular mechanisms underlying obesityinduced arterial stiffness. Am J Physiol Regul Integr Comp Physiol. 2018; 314(3):R387-R398.

\section{Fontes de financiamento}

$\mathrm{O}$ presente estudo foi financiado pelo CNPq (Processo 433898/2018-06)

\section{Vinculação acadêmica}

Não há vinculação deste estudo a programas de pós-graduação.

18. Saladini F, Palatini P. Arterial distensibility, physical activity, and the metabolic syndrome. Curr Hypertens Rep. 2018; 20(5):39.

19. Wang X, Khalil RA. Matrix metalloproteinases, vascular remodeling, and vascular disease. Adv Pharmacol. 2018; 81(1):241-330.

20. Lee D, Sui X, Church TS, Lavie CJ, Jackson AS, Blair SN. Changes in fitness and fatness on the development of cardiovascular disease risk factors: hypertension, metabolic syndrome, and hypercholesterolemia. J Am Coll Cardiol. 2012; 59(7):665-72.

21. Stokes KY, Calahan L, Russell JM, Gurwara S, Granger DN. Role of platelets in hypercholesterolemia-induced leukocyte recruitment and arteriolar dysfunction. Microcirculation. 2006; 13(5):377-88.

22. Stokes KY, Calahan L, Hamric CM, Russell JM, Granger DN. CD40/CD40L contributes to hypercholesterolemia-induced microvascular inflammation. Am J Physiol Heart Circ Physiol. 2009; 296(3):H689-H697.

23. Xu Y, Arai H, Murayama T, Kita T, Yokode M. Hypercholesterolemia contributes to the development of atherosclerosis and vascular remodeling by recruiting bone marrow-derived cells in cuff-induced vascular injury. Biochem Biophys Res Commun. 2007; 363(3):782-7.

24. Lewis GF, Rader DJ. New insights into the regulation of HDL metabolism and reverse cholesterol transport. Circ Res. 2005; 96(12):1221-32.

25. Karlson BW, Palmer MK, Nicholls SJ, Lundman P, Barter PJ. Doses of rosuvastatin, atorvastatin and simvastatin that induce equal reductions in LDL-C and non-HDL-C: results from the VOYAGER meta-analysis. Eur J Prev Cardiol. 2016; 23(7):744-7.

26. Wang CY, Liu PY, Liao JK. Pleiotropic effects of statin therapy: molecular mechanisms and clinical results. Trends Mol Med. 2008; 14(1):37-44.

27. Brooks SD, DeVallance E, d'Audiffret AC, Frisbee SJ, Tabone LE, Shrader $\mathrm{CD}$, et al. Metabolic syndrome impairs reactivity and wall mechanics of cerebral resistance arteries in obese Zucker rats. Am J Physiol Heart Circ Physiol. 2015; 309(11):H1846-H1859.

28. Izidoro-Toledo TC, Guimaraes DA, Belo VA, Gerlach RF, Tanus-Santos JE. Effects of statins on matrix metalloproteinases and their endogenous inhibitors in human endothelial cells. Naunyn Schmiedebergs Arch Pharmacol. 2011; 383(6):547-54.

29. Luan Z, Chase AJ, Newby AC. Statins Inhibit Secretion of metalloproteinases-1, -2, -3, and -9 From Vascular Smooth Muscle Cells and Macrophages. Arterioscler Thromb Vasc Biol. 2003; 23(5):769-75.

30. Rupérez M, Rodrigues-Díez R, Blanco-Colio LM, Sánchez-López E, Rodríguez-Vita J, Esteban V, et al. HMG-CoA Reductase Inhibitors Decrease Angiotensin II-induced Vascular Fibrosis: Role of RhoA/ROCK and MAPK Pathways. Hypertension. 2007; 50(2):377-83.

31. Bruder-Nascimento T, Callera GE,MontezanoAC,ChantemeleEB, Tostes RC, Touyz RM. Atorvastatin inhibits pro-inflammatory actions ofaldosterone in vascularsmooth muscle cells by reducing oxidative stress. Life Sci. 2019; 221(1):29-34.

32. Liping Z, Xiufang L, Tao Y, Baomin Z, Houshuai T. Efficacy comparison of rosuvastatin and atorvastatin in the treatment of atherosclerosis and drug safety analysis. Pak J Pharm Sci. 2018; 31(5(Special)):2203-8.

33. Kumar B, Shah MAA, Kumar R, Kumar J, Memon A. Comparison of atorvastatin and rosuvastatin in reduction of inflammatory biomarkers in patients with acute coronary syndrome. Cureus. 2019; 11(6):e4898. 
34. Yamada Y, Takeuchi S, Yoneda M, Ito S, Sano Y, Nagasawa K, et al. Atorvastatin reduces cardiac and adipose tissue inflammation in rats with metabolic syndrome. Int J Cardiol. 2017; 240(1):332-8.

35. Taylor BA, Phillips SJ. Detection of obesity QTLs on mouse chromosomes 1 and 7 by selective DNA pooling. Genomics. 1996; 34(3):389-98.

36. Fernandes SAT, Natali AJ, da Matta SLP, Teodoro BG, Franco FSC, Laterza $M C$, et al. Effect of hyperlipidic diet and aerobic training on atherosclerosis in apoE-/- mice. Rev Bras Med Esporte. 2013; 19(6):436-41.

37. Costa RM, Neves KB, Mestriner FL, Louzada-Junior P, Bruder-Nascimento T, Tostes RC. TNF-? induces vascular insulin resistance via positive modulation of PTEN and decreased Akt/eNOS/NO signaling in high fat diet-fed mice. Cardiovasc Diabetol. 2016;15(1):119.

38. Parlee SD, Lentz SI, Mori H, MacDougald OA. Quantifying size and number of adipocytes in adipose tissue. Methods Enzymol. 2014; 537(1):93-122.

39. Silva JF, Correa IC, Diniz TF, Lima PM, Santos RL, Cortes SF, et al. Obesity, inflammation, and exercise training: relative contribution of iNOS and eNOS in the modulation of vascular function in the mouse aorta. Front Physiol. 2016; 7 (eCollection 2016):386.

40. Leopoldo AS, Lima-Leopoldo AP, Nascimento AF, Luvizotto RAM, Sugizaki MM, Campos DHS, et al. Classification of different degrees of adiposity in sedentary rats. Braz J Med Biol Res. 2016; 49(4):e5028

41. Rezende R, Santos C, Dubow C, Vargas SC, Pohl HH, Paiva DN. Effects of physical exercise on insulin resistance to obese individuals. Cinergis. 2016; 17(3):245-9.

42. Mullugeta Y, Chawla R, Kebede T, Worku Y. Dyslipidemia associated with poor glycemic control in type 2 diabetes mellitus and the protective effect of metformin supplementation. Indian J Clin Biochem. 2012; 27(4):363-9.

43. Fonseca VA, Lovre D. Diabetes-related dyslipidemia and cardiovascular events. Am J Med Sci. 2017; 354(2):103-4.

44. Silva JM. The efficacy of Atorvastatin in correcting the lipid profile-An open evaluation study. Medicina Interna. 2002; 9(2):68-75.

45. Parhofer KG, Barrett PH, Schwandt P. Atorvastatin improves postprandia lipoprotein metabolism in normolipidemlic subjects. J Clin Endocrinol Metab. 2000; 85(11):4224-30.

46. Kissebah AH, Vydelingum N, Murray R, Evans DJ, Hartz AJ, Kalkhoff RK, et al. Relation of body fat distribution to metabolic complications of obesity. J Clin Endocrinol Metab. 1982; 54(2):254-60.

47. Taylor AE, Ebrahim S, Ben-Shlomo Y, Martin RM, Whincup PH, Yarnell JW, et al. Comparison of the associations of body mass index and measures of central adiposity and fat mass with coronary heart disease, diabetes, and all-cause mortality: a study using data from 4 UK cohorts. Am J Clin Nutr. 2010; 91(3):547-56.

48. Carvalho MHC, Colaço AL, Fortes ZB. Cytokines, endothelial dysfunction, and insulin resistance. Arq Bras Endocrinol Metab. 2006; 50(2):304-12.

49. Karczewski J, Sledzinska E, Baturo A, Jonczyk I, Maleszko A, Samborski P, et al. Obesity and Inflammation. Eur Cytokine Netw. 2018; 29(3):83-94.

50. Suzuki M, Kakuta H, Takahashi A, Shimano H, Tadalida K, Yokoo T, et al Effects of atorvastatin on glucose metabolism and insulin resistance in kk/ay mice. J Atheroscler Thromb. 2005; 12(2):77-84.

51. Huptas S, Geiss HC, Otto C, Parhofer KG. Effect of atorvastatin (10 mg/ day) on glucose metabolism in patients with the metabolic syndrome. Am J Cardiol. 2006; 98(1):66-9.

52. Mulvany MJ. Vascular remodelling of resistance vessels: can we define this? Cardiovasc Res. 1999; 41(1):9-13.

53. Galic S, Oakhill JS, Steinberg GR. Adipose tissue as an endocrine organ. Mo Cell Endocrinol. 2010; 316(2):129-39.

54. Heber D. An integrative view of obesity. Am J Clin Nutr. 2010 91(1):280S-283S

55. Marginean CO, Melit LE, Hutanu A, Ghiga DV, Sasaran MO. The adipokines and inflammatory status in the era of pediatric obesity. Cytokine. 2020 126(1):154925.

56. Miao CY, Li ZY. The Role of Perivascular Adipose Tissue in Vascular Smooth Muscle Cell Growth. Br J Pharmacol. 2012; 165(3):643-58.

57. Matsuzawa Y. Adipocytokines and metabolic syndrome. Semin Vasc Med 2005; 5(1):34-9.

58. Takaoka M, Suzuki H, Shioda S, Sekikawa K, Saito Y, Nagai R, et al. Endovascular injury induces rapid phenotypic changes in perivascular adipose tissue. Arterioscler Thromb Vasc Biol. 2010; 30(8):1576-82.

59. Blomkalns AL, Gavrila D, Thomas M, Neltner BS, Blanco VM, Benjamin SB, et al. CD14 directs adventitial macrophage precursor recruitment: role in early abdominal aortic aneurysm formation. J Am Heart Assoc. 2013; 2(1):e000065.

60. Ozen G, Daci A, Norel X, Topal G. Human perivascular adipose tissue dysfunction as a cause of vascular disease: focus on vascular tone and wall remodeling. Eur J Pharmacol. 2015; 766(1):16-24

61. Duan S, Zhang Y, Wu S, Jiang L, Zhang J, Gan Y, et al. Atorvastatin attenuates inflammatory infiltration and vascular remodeling in lung of hypercholesterolemia rabbits. Exp Lung Res. 2010; 36(10):573-92. 